\title{
Light curves and colours of the faint Uranian irregular satellites Sycorax, Prospero, Stephano, Setebos, and Trinculo ${ }^{\star} \star \star$
}

\author{
M. Maris ${ }^{1}$, G. Carraro ${ }^{2,3}$, and M. G. Parisi ${ }^{4,5,6}$ \\ 1 INAF - Osservatorio Astronomico di Trieste, via G.B. Tiepolo 11, 34131 Trieste, Italy \\ e-mail: maris@oats.inaf.it \\ 2 Dipartimento di Astronomia, Università di Padova, Vicolo Osservatorio 2, 35122 Padova, Italy \\ e-mail: giovanni.carraro@unipd.it \\ 3 Andes Prize Fellow, Universidad de Chile and Yale University \\ 4 Departamento de Astronomía, Universidad de Chile, Casilla 36-D, Santiago, Chile \\ e-mail: gparisi@das.uchile.cl \\ 5 Member of the Centro de Astrofisica, Fondo de Investigacion Avanzado en Areas Prioritarias (FONDAP), Chile \\ ${ }^{6}$ Member of the Consejo Nacional de Investigaciones Cientificas y Tecnicas (CONICET), Argentina
}

Received 13 December 2006 / Accepted 17 April 2007

\section{ABSTRACT}

\begin{abstract}
Context. After the work of Gladman et al. (1998, Nature, 392, 897), it is now assessed that many irregular satellites are orbiting around Uranus.

Aims. Despite many studies performed in past years, very little is know about the light-curves of these objects and inconsistencies are present between colours derived by different authors. This situation motivated our effort to improve both the knowledge of colours and light curves.

Methods. We present and discuss, the time series observations of Sycorax, Prospero, Stephano, Setebos, and Trinculo, five faint, irregular satellites of Uranus, which were carried out at VLT, ESO Paranal (Chile) on the nights between 29 and 30 July, 2005 and 25 and 30 November, 2005.

Results. We derive light curves for Sycorax and Prospero and colours for all of these these bodies.

Conclusions. For Sycorax we obtain colours $B-V=0.839 \pm 0.014, V-R=0.531 \pm 0.005$, and a light-curve which is suggestive of a periodical variation with period $\approx 3.6 \mathrm{~h}$ and amplitude $\approx 0.067 \pm 0.004 \mathrm{mag}$. The periods and colours we derive also for Sycorax are in agreement with our previous determination in 1999 using NTT. We also derive a light-curve for Prospero which suggests an amplitude of about 0.2 mag and a periodicity of about $4 \mathrm{~h}$. However, the sparseness of our data, prevents a more precise characterization of the light-curves, and we can not determine whether they are one-peaked or two-peaked. Hence, these periods and amplitudes have to be considered preliminary estimates. As for Setebos, Stephano, and Trinculo the present data do not allow us to derive any unambiguous periodicity, despite the fact that Setebos displays a significant variability with amplitude about as large as that of Prospero. Colours for Prospero, Setebos, Stephano, and Trinculo are in marginal agreement with the literature.
\end{abstract}

Key words. planets and satellites: general - methods: observational - methods: data analysis - methods: statistical methods: numerical

\section{Introduction}

In recent years many irregular satellites have been discovered around Uranus (Gladman et al. 1998; Kavelaars et al. 2004; Gladman et al. 2000; Sheppard et al. 2005). Irregular satellites are those planetary satellites on highly elliptic and/or highly inclined (even retrograde) orbits with a large semi-major axis. These objects cannot have formed by circumplanetary accretion like the regular satellites, but they are likely products of captures from heliocentric orbits, probably in association with the planet formation itself (Greenberg 1976; Morrison \& Burns 1976; Morrison et al. 1977; Jewitt \& Sheppard 2005). It is possible for an object circling about the Sun to be temporarily trapped by a planet (Heppenheimer 1975; Greenberg 1976; Morrison \& Burns 1976, to cite only some). But to turn a

\footnotetext{
* Based on observations with the ESO Very Large Telescope + FORS2 at the Paranal Observatory, Chile, under program 075.C-0023.

$\star \star$ Table 1 is only available in electronic form at http://www. aanda.org
}

temporary capture into a permanent one requires a source of dissipation of orbital energy and that particles remain inside the Hill sphere long enough for the capture to be effective (Pollack et al. 1979). Otherwise, the trapped object will escape within at most a few hundred orbits (Byl \& Ovenden 1975; Heppenheimer 1975; Heppenheimer \& Porco 1977; Pollack et al. 1979). During the planet formation epoch several mechanisms may have operated, some of which have the potential to be active even after this early epoch. They fall mainly into few categories: collisional interactions (Colombo \& Franklin 1971), pull-down capture (Heppenheimer \& Porco 1977), gas drag (Pollack et al. 1979), four-bodies interactions in the reference frame of the the Sun-Planet system either between the captured body and a large regular satellite of the planet (Tsui 2000), or between the two components of a binary object leading to an exchange reaction where one of the components of the binary is captured and the other is ejected from the system (Agnor \& Hamilton 2006).

Collisional capture, the so called break-up process, leads to the formation of dynamical groupings. The resulting fragments 
of the progenitor body after a break-up will form a population of irregular satellites expected to have similar composition, i.e., similar colours, and irregular surfaces. Large temporal variations in the brightness of irregular satellites are expected from rotating bodies of highly elongated shapes and/or irregular surfaces consistent with a collision fragment origin.

Gas drag is expected to occur in the environment of the protoplanetary nebula (Byl \& Ovenden 1975; Horedt 1976; Heppenheimer \& Porco 1977; Pollack et al. 1979) and it may be that the origin of dynamical families of fragments. In this case fragments would be produced by the hydrodynamical breaking of the intruding body into smaller chunks in the case that they exceed the tensile strength of the entering body (Pollack et al. 1979). The gravitational attraction of fragments prevents them from escaping, in general the hydrodynamical pull being not larger than self-gravity, but a small impact of a $\sim 1 \mathrm{~km}$ size object, likely common in the nebula environment, would be sufficient to disperse them without introducing a further fragmentation (Pollack et al. 1979). A specific prediction of this scenario is the production of fragments with a more regular/round surface than in the break-up process, leading to a light-curve with low amplitude variations (Pollack et al. 1979).

On the contrary, if pull-down capture, four-bodies interactions or exchange reactions are the dominant causes of formation of the irregular satellites, each object would be the result of an independent capture event. In this case, no obvious correlation between dynamical properties, colours, and light-curves would be expected.

Casting light on these scenarios, colours, and light curves are very important, since they would allow one to discriminate between collisional or non-collisional origin for irregular satellites. Theories of irregular satellite capture have lacked many constraints. However, the rapidly-growing number of known irregular satellites is now providing new insights on the processes of planet formation and satellite capture.

A possible origin of the large obliquity of Uranus is a giant impact event between the planet and an Earth-sized planetesimal, occurred at the end of the epoch of accretion (Slattery et al. 1992; Parisi \& Brunini 1997). The dynamical and physical properties of the Uranian irregular satellites may shed light on their capture mechanism and may also constrain the mechanism leading to the peculiar tilt of the planet's rotation axis (Brunini et al. 2002; Parisi et al. 2007). For example, significant fluctuations have been observed in the Caliban light-curve for which data are consistent with a light-curve with amplitude $A_{\text {Caliban }}=0.12 \pm 0.01 \mathrm{mag}$ and a most probable period of $\approx 2.7 \mathrm{~h}$ as in the Sycorax light curve, $A_{\text {Sycorax }}=0.032 \pm 0.008$ mag with either a period of $\approx 4.1 \mathrm{~h}$ or $\approx 3.7 \mathrm{~h}$ (Maris et al. 2001). In this regard Romon et al. (2001) report discrepancies in the spectrum they possibly attributed to rotational effects. All of this seems to support the idea of a collisional scenario. However, the existence of a dynamical grouping (Kavelaars et al. 2004; Sheppard et al. 2005 ) is still debated on the light of the colour determination of Grav et al. (2004b). Regrettably, there seems to be a lack of consistency between $B-V$ and $V-R$ colours of different authors for Sycorax and Caliban (Maris et al. 2001; Rettig et al. 2001; Romon et al. 2001; Grav et al. 2004a). This may be ascribed to systematic differences in the photometry and accompanying calibration or, at least for Caliban, to rotational effects.

In an attempt to improve on the situation, in this paper we present and discuss new observations of five irregulars of Uranus, Sycorax, Prospero, Setebos, Stephano, and Trinculo, obtained with the ESO Very Large Telescope on Cerro Paranal, Chile.
The paper is organised as follows: Sect. 2 describes observations and data reduction, light-curves are discussed in Sect. 3, while Sect. 4 presents the satellites' colours. The conclusions are reported in Sect. 5.

\section{Observations and data reduction}

We observed the irregular satellites Sycorax, Prospero, Stephano, Trinculo and Setebos with the FORS2 camera (Appenzeller et al. 1998) at the focus of VLT Antu telescope in Paranal, Chile, in the two consecutive nights of July 28 and 29, 2005.

We used the standard FORS2 $B, V, R, I$ filters ${ }^{1}$, which are very close to the Bessel system. In particular, the effective wavelength, $\lambda_{\mathrm{eff}}$, and FWHM, $\Delta \lambda$, for the filters reported by ESO are $\lambda_{\mathrm{eff}, B}=0.429 \mu \mathrm{m}, \Delta \lambda_{B}=0.0880 \mu \mathrm{m}$ for $B ; \lambda_{\mathrm{eff}, V}=0.554 \mu \mathrm{m}$, $\Delta \lambda_{V}=0.1115 \mu \mathrm{m}$ for $V ; \lambda_{\mathrm{eff}, R}=0.655 \mu \mathrm{m}, \Delta \lambda_{R}=0.1650 \mu \mathrm{m}$ for $R$; and $\lambda_{\mathrm{eff}, I}=0.768 \mu \mathrm{m}, \Delta \lambda_{I}=0.1380 \mu \mathrm{m}$ for $I$. Stephano and Trinculo were observed in the same frames so that we observe five objects with just four sequences. For this reason Stephano $R 1, R 2, \ldots, V 1, V 2, \ldots$, frames correspond to Trinculo $R 1, R 2, \ldots, V 1, V 2, \ldots$, frames. Each object was observed in consecutive sequences of frames. After the end of the sequence for a given object the telescope switched to the sequence of another object. Ideally, colours would have to be calculated by combining magnitudes from frames in the same sequence, in order to limit the rotational effects. For each sequence, pointing of the telescope and orientation of the camera were kept fixed. During the acquisition of each frame the telescope was tracked at the same rate the target, while the telescope was reset at the default pointing at the beginning of each frame in the sequence. However, given the slow proper motion and the short exposures, the effect of differential tracking on background stars was negligible, background stars do not appear elongated. Interruptions due to a ToO, mid-night calibrations, and some minor problem prevent us from keeping the same sequences both nights. Both nights were photometric, with average seeing $\approx 1.1$ arcsec. FORS2 is equipped with a mosaic of two $2 \mathrm{k} \times 4 \mathrm{k}$ MIT CCDs (pixel size of $15 \times 15$ micron) with a pixel scale, with the default 2-by-2 binning, of 0 '.25/pixel. The satellite and Landolt (1992) standard stars were centred in CCD \#1. Pre-processing of images, which includes bias and flat field corrections, were done using standard $\mathrm{IRAF}^{2}$ routines. Aperture photometry was then extracted using the IRAF tool QPHOT, both for the standard stars and the satellite, using a handful of bright field stars to estimate the aperture correction. The resulting corrections were small, going from 0.06 to 0.25 in all filters. A series of $R$ exposures were taken with the aim to construct a light curve and search for some periodicity. A few $B, V$, and $I$ exposures were taken as well to constrain the satellites' colours. The calibration was derived from a grand total of 30 standard stars per night in the PG0231+051, SA92, PG2331+055, MARK A, SA111, PG1528+062, and PG1133+099 Landolt (1992) fields. The two nights showed identical photometric conditions, and therefore a single photometric solution was derived for the whole observing run

$M=m_{\mathrm{inst}}+\alpha_{\mathrm{m}}-\beta_{\mathrm{m}} \cdot$ Airmass;

where $m_{\text {inst }}=b, v, r$ or $i$ are the instrumental magnitudes, $M=B, V, R$, or $I$ are the reduced magnitudes, Airmass is the

\footnotetext{
1 See http://www.eso.org/instruments/fors/inst/Filters/ for further details.

2 IRAF is distributed by NOAO, which are operated by AURA under cooperative agreement with the NSF.
} 


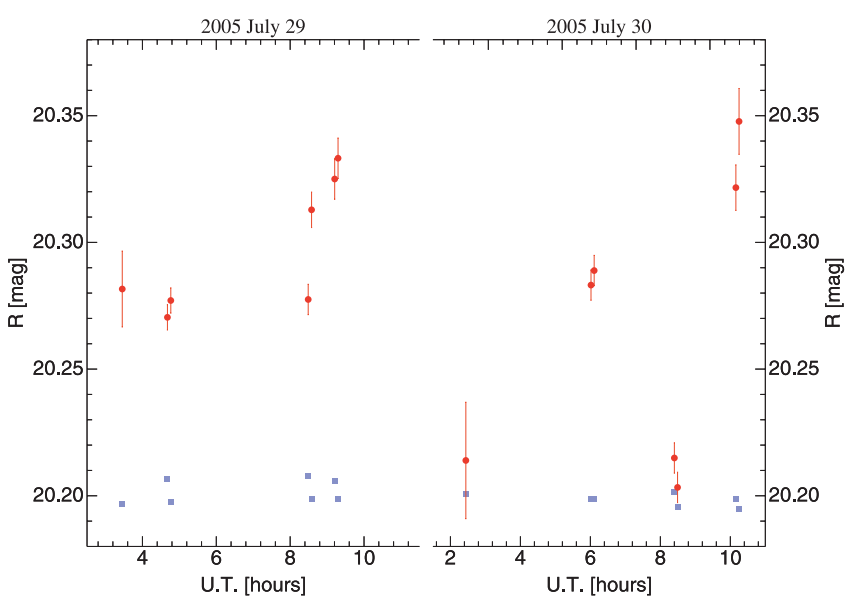

Fig. 1. Light curves in $R$ for Sycorax. Data are for the nights of 2005, July 29th (left) and 30th (right). Squares in gray represent measurements of magnitudes for a common field star of similar magnitude. To avoid confusion error bars for the field star are not reported and the averaged magnitude is shifted. (This figure is available in color in electronic form.)

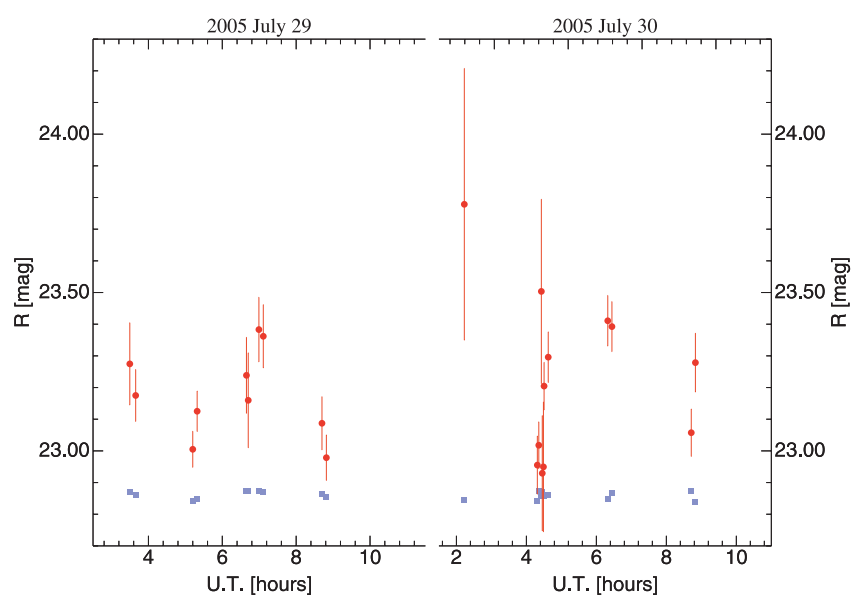

Fig. 2. Light curves $R$ for Prospero. (See Fig. 1 for comments.)

airmass and $\alpha_{\mathrm{m}}$ and $\beta_{\mathrm{m}}$ are the calibration coefficients. We obtain $\alpha_{\mathrm{m}}=2.332,2.864,3.112,2.546$, and $\beta_{\mathrm{m}}=0.269,0.177,0.147$. 0.150 respectively for the $B, V, R$, and $I$ bands. No colour correction have been applied due to the very small colour term. A few additional observations of Prospero were acquired on the nights of November 22 and 25, 2005 in compensation at the ToO. We reduced the data in the same way as in the July run, but obtained an independent calibration, being $\alpha_{\mathrm{m}}=2.318,2.879,3.007$, 2.529 for the $B, V, R$, and $I$ bands, respectively, which is very similar to the July one. The list of measures for the five satellites is in Table 1. The table reports the reduced magnitudes, errors and exposure times. The shortest exposures have been acquired to improve frame centering nevertheless we report magnitudes from these frames too. The time column refers to the starting time of each exposure. No corrections for light travel times was applied to these data.

\section{Light curves}

Figures 1-5 presents $R$ light curves respectively for Sycorax, Prospero, Setebos, Stephano and Trinculo for the July 2005 nights. Each plot is splitted in two subpanels, the left for the July 29th and the right for July 30th. Squares in gray represents

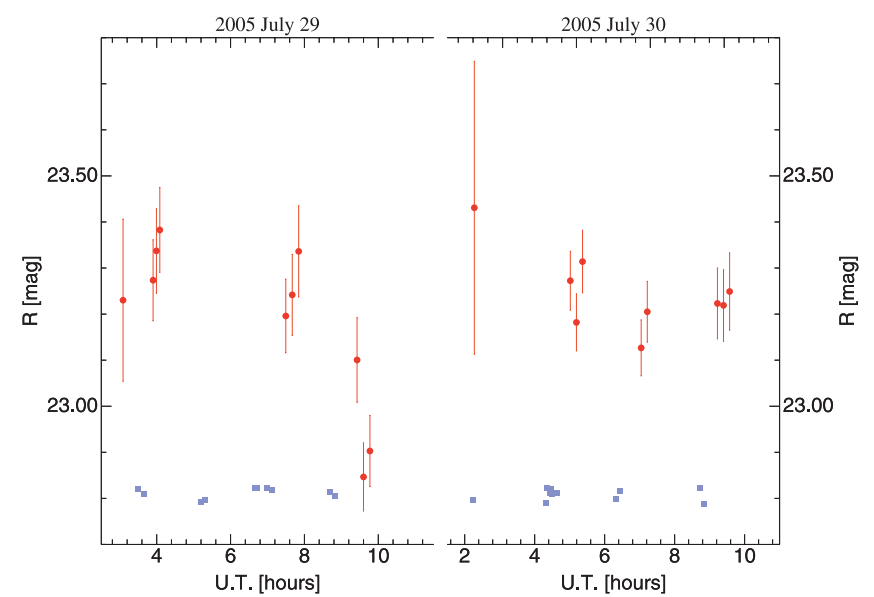

Fig. 3. Light curves of Setebos. (See Fig. 1 for comments.)

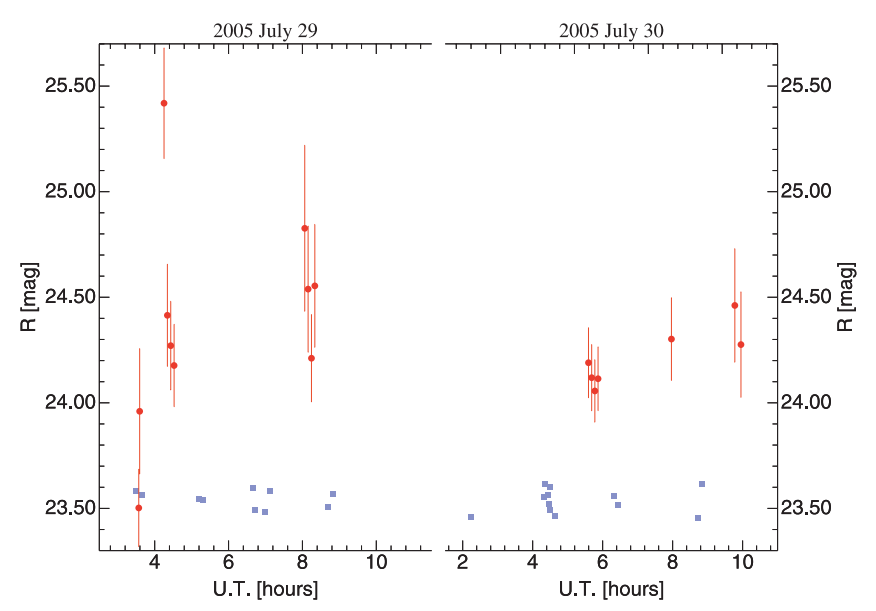

Fig. 4. Light curves $R$ for Stephano. (See Fig. 1 for comments.)

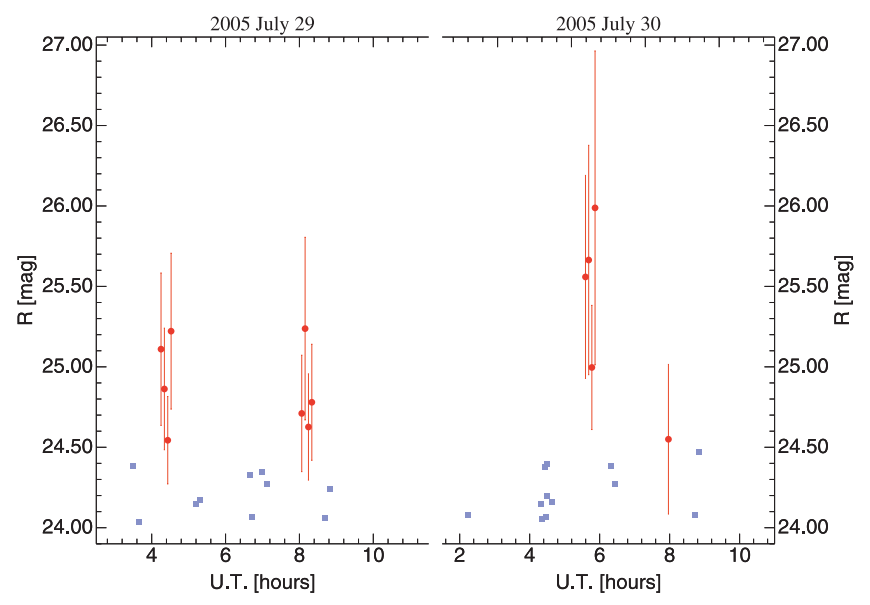

Fig. 5. Light curves $R$ for Trinculo. (See Fig. 1 for comments.)

the measurements of a common field star with similar magnitude. To avoid confusion, error bars for the field star are not reported and the averaged magnitude is shifted. For the same reason the few data obtained in November 2005 for Prospero listed in the Table 1 are not plotted in Fig. 2.

We analyse magnitude fluctuations in the light-curves trying to assess first of all whether the detected variations may be ascribed to random errors, Sect. 3.1, instabilities in the zero point of the calibration, Sect. 3.2, or to the effect of unresolved 
Table 2. Testing against random fluctuations. Columns $2-3 \chi^{2}$ and significativity level (SL) assuming constant signal; $4-5 \chi^{2}$ and SL assuming linear time dependence; 6-7 maximum amplitude and false allarm probability assuming periodical signal. Note that in all the cases a low probability (either SL or $P_{\mathrm{FA}}$ ) denotes a high level of confidence in excluding noise fluctuations.

\begin{tabular}{lcccccc}
\hline \hline & \multicolumn{9}{c}{ Hypothesis H0 } \\
Object & $\chi_{\text {const }}^{2}$ Constant & SL & $\chi_{\text {linear }}^{2}$ & SL & $A_{\text {max }}$ & $P_{\text {FA }}$ \\
\hline Sycorax & 557 & $<1 \times 10^{-9}$ & 437 & $<1 \times 10^{-9}$ & 0.07 & $<1 \times 10^{-8}$ \\
Prospero & 92.7 & $1 \times 10^{-7}$ & 85.2 & $1 \times 10^{-6}$ & 0.22 & $<3 \times 10^{-6}$ \\
Stephano & 45.3 & $4 \times 10^{-4}$ & 44.9 & $3 \times 10^{-4}$ & 0.36 & 0.22 \\
Setebos & 59.2 & $3 \times 10^{-5}$ & 59.2 & $2 \times 10^{-5}$ & 0.19 & $7 \times 10^{-3}$ \\
Trinculo & 8.83 & $8 \times 10^{-1}$ & 8.44 & $8 \times 10^{-1}$ & 0.42 & 0.44 \\
\hline
\end{tabular}

background objects Sect. 3.3. In case the variability has been judged significant, attempt to estimate the amplitude and the period, Sect. 3.4. We use both analytical methods and a Monte Carlo (MC) - Bootstrap technique.

\subsection{Testing against random fluctuations}

The results of a $\chi^{2}$ test performed on $V, R$, and in some cases, $B$ and $I$ measures are reported in the first two columns of Table 2 (similar results are obtained by a bootstrap on the data). In this test the hypothesis $H_{0}$ to be disproved is that the data are compatible with a constant signal (different from filter to filter) with random errors as the sole cause of brightness fluctuations. The table shows that this hypothesis may be discarded for Prospero and Sycorax with a very high level of confidence. As for Stephano and Setebos the level is lower but still significant, whereas for Trinculo the hypothesis can not be discarded at all. Before considering the case for a periodic variation, the case for a systematic trend in the brightness is considered, since the irregular sampling in time prevents the application of robust de-trendization techniques. As evident from the table, even this case can be excluded by the present data at a level of confidence similar to the constant case.

\subsection{Field stars analysis}

In order to assess the level of calibration accuracy in an independent manner, several field stars having magnitudes encompassing those of the irregular satellites and common to each frame in both nights have been measured in the same way as the satellites (see Carraro et al. 2006, for an example of the adopted technique). As shown in Figs. 1-5 field stars are characterised by narrower fluctuations than satellites. A more quantitative test is obtained by taking a set of variability indicators by measuring the variability of field stars and satellites and comparing them. This is done in Fig. 6 where two variability indicators, the peakto-peak variation (top) and the rms (bottom), for satellites (red spots) and field stars (light blue asterisks) are plotted against the $R$ magnitude of the objects. The first important thing to note is the good consistency between the two indicators. While it is evident that the variability of Sycorax, Prospero and Setebos is above the level of variability of field stars, the same is not true for Stephano and Trinculo. In addition, a test for the correlation of, for example Sycorax or Prospero and the related field stars shows that they are not significantly correlated. As an example the correlation coefficient between Sycorax and three field stars is $C_{\text {Sycorax,s }}=-0.36,-0.08$ and -0.53 . The probability
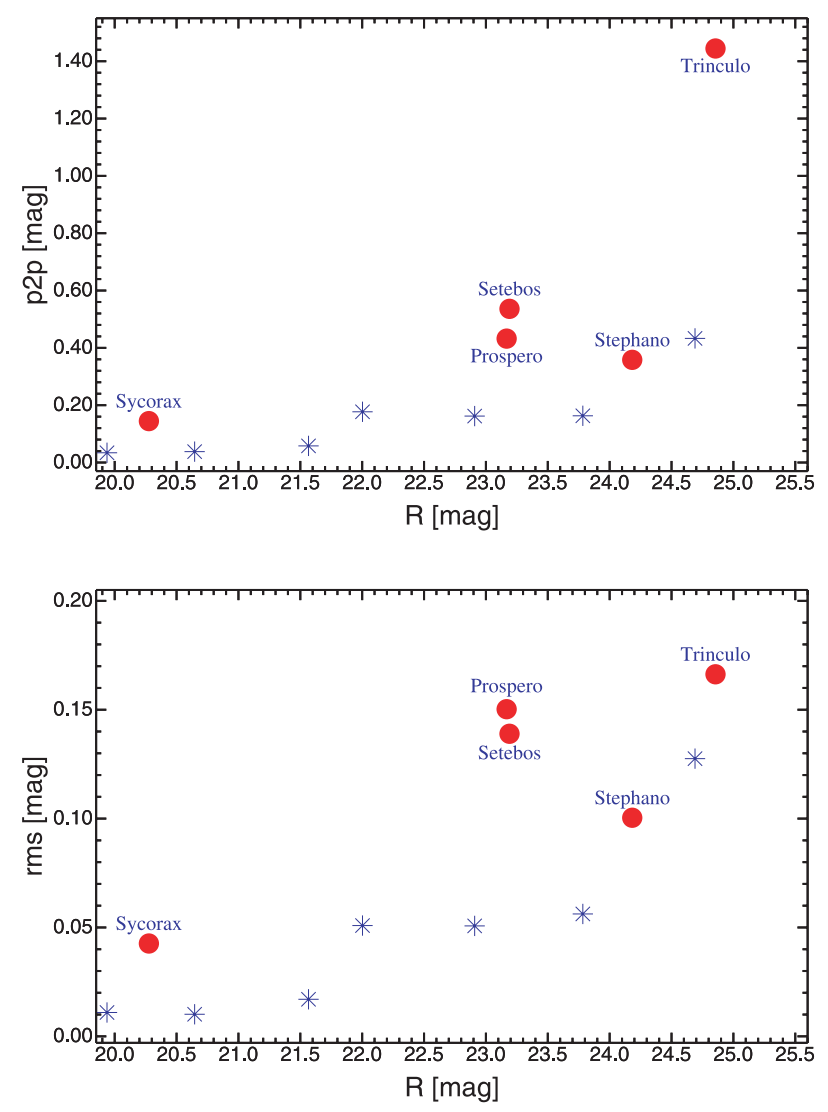

Fig. 6. Variability of the $R$ light curves for irregular satellites (red circles) and field stars (light blue asterisks) as a function of their magnitude. Top frame for the peak-to-peak variation, bottom frame for rms of the variation. (This figure is available in color in electronic form.)

that this level of correlation can be reached by chance even if their time series are not correlated are respectively $27 \%, 78 \%$, $15 \%$, while for Prospero $C_{\text {Prospero,s }}=-0.10,0.22$, and -0.12 with probabilities respectively of $64 \%, 36 \%$, and $59 \%$. In addition, even assuming a correlation between field stars fluctuations and satellite fluctuations, it would explain only a small fraction of the satellites' variability. Indeed, variances of field stars accounts for $0.02 \%-6 \%$ of the Sycorax variance, $6 \%-9 \%$ of the Prospero variance, $12 \%-18 \%$ of the Setebos variance, $2 \%-14 \%$ of Stephano or Trinculo variances. In conclusion, field stars' variability, connected to calibration instabilities, can not account for most of the variability of at least Sycorax, Prospero, and Setebos, while as evident from Fig. 6 Stephano and Trinculo would have to be considered more cautiously.

\subsection{Unresolved background objects}

Unresolved objects in the background may affect photometry. A quick-look at the frames ${ }^{3}$ shows that in general, the satellites pass far enough from background objects to allow a proper separation of their figures. It can be considered also the case in which a satellite crosses the figure of an undetected faint object in the background producing a fake time variability of the satellite light-curve. It is quite simple to compute the upper limit for the magnitude of a background object, $R_{\mathrm{bck}}$, able to produce the observed variations of magnitude for these satellites.

\footnotetext{
3 A sequence of the observed frames for Prospero has been already published in Parisi et al. (2007).
} 
The result is $R_{\text {bck }} \leq 23.6 \mathrm{mag}, 24.7 \mathrm{mag}, 25 \mathrm{mag}$, $25.5 \mathrm{mag}$, and $25.5 \mathrm{mag}$, respectively for Sycorax, Prospero, Setebos, Stephano, and Trinculo. Those magnitudes are within the detection limit for Sycorax and Prospero, near the detection limit for Setebos and marginally outside the detection limit for Stephano, and Trinculo. In conclusion, at least for Sycorax and Prospero, we can be confident that the background is not important.

\subsection{Looking for amplitudes and periodicities}

Given the sparseness of our data it is not easy to asses safely the shapes, the amplitudes and the periods of our light-curves, despite at least for Sycorax, Prospero and probably Setebos a significant variability is present. However, we think it is important to attempt a recover of such information, at least as a step towards planning of more accurate observations.

Constraints on the amplitude for the part of light curves sampled by our data may be derived from the analysis of the peakto-peak variation. After excluding data with exposure times below $100 \mathrm{~s}$ we evaluate peak-to-peak variations for $R, \Delta R_{\mathrm{p} 2 \mathrm{p}}$. Denoting with $A_{R}$ the amplitude of the light-curve we may assume $A_{R} \approx \Delta R_{\mathrm{p} 2 \mathrm{p}} / 2$. To cope with the random noise, $\Delta R_{\mathrm{p} 2 \mathrm{p}}$ have been evaluated by MonteCarlo, simulating the process of $\Delta R_{\mathrm{p} 2 \mathrm{p}}$ evaluation assuming that the random errors of the selected data are normally distributed. We obtain $A_{R, \text { Sycorax }} \gtrsim 0.07 \pm 0.01 \mathrm{mag}$, $A_{R \text {,Prospero }} \gtrsim 0.27 \pm 0.04 \mathrm{mag}$, and $A_{R \text {,Setebos }} \gtrsim 0.31 \pm 0.05 \mathrm{mag}$. Where the $\gtrsim$ symbol is used because the $A_{R} \approx \Delta R_{\mathrm{p} 2 \mathrm{p}} / 2$ relation is strictly valid only if the true minima and maxima of light-curves are sampled, a condition which we are not sure to have fullfilled. Another order of magnitude estimate of the amplitudes is based on the analysis of their $\operatorname{rms}, \operatorname{std}\left(R_{t}\right)$. It is easy to realize that for any periodical light-curve of amplitude $A_{R}$ then $\operatorname{std}\left(R_{t}\right) \approx f_{\text {Dump }} A_{R}$. Where $f_{\text {Dump }}>0$ is a factor which depends both on the sampling function and the shape of signal. Assuming a sinusoidal signal sampled sufficiently well, $f_{\text {Dump }} \approx 1.39-1.46$, giving for Sycorax $A_{R, \text { Sycorax }} \approx 0.06 \mathrm{mag}$, while for Prospero and Setebos $A_{R \text {,Prospero }} \approx A_{R \text {, Setebos }} \approx 0.2 \mathrm{mag}$.

We then consider the case for a periodical variation in our light-curves by attempting first to search for the presence of periodicities in the hypothesys of a sinusoidal time dependence, and in case of a positive answer, assessing the most likely sinusoidal amplitude. To cope with the limited amount of data increasing both the sensitivity to weak variations and the discrimination power against different periods, we fit the same sinusoidal dependence on $V, R$, and in some cases $B$ and $I$ measures, assuming that colours are not affected by any significant rotation effect. In short the model to be fitted is

$M_{f}=A \cos \left(\frac{2 \pi}{P}(t-\tau)+\phi\right)+M_{f, 0}$,

where $f=B, V, R$, and $I$, indicates the filter, $M_{\mathrm{f}}$ the measured magnitudes for that filter, $M_{\mathrm{f}, 0}$ the averaged magnitude for the filter $f, A$ is the amplitudes $\phi$ the phase and $\tau$ an arbitrary origin in time. It has to be noted that phase-angle effects are not considered here. The reason is that no sure dependence of the magnitude on the phase-angle has been established so far for these bodies. On the other hand, the variation of the phase angles over two consecutive nights is just about 2.4 arcmin. Consequently, we expect the phase-angle effect to be quite negligible. As widely discussed in literature, the problem of searching for periodicities by fitting a model with a sinusoidal time dependence is equivalent to the analysis of the periodogram for the given data set (Lomb 1976; Scargle 1982; Cumming et al. 1999; Cumming 2004, just to cite some). The Lomb and Scargle (hereinafter LS) periodogram is the most commonly used version. In this view, a better formulation of the problem is obtained expressing the model in the following form:

$M_{f}=A_{\mathrm{c}} \cos (\omega(t-\tau))+A_{\mathrm{s}} \sin (\omega(t-\tau))+M_{f, 0}$

where $\omega=\frac{2 \pi}{P}, A_{\mathrm{c}}$ and $A_{\mathrm{s}}$ co-sinusoidal and sinusoidal amplitudes related to $A$ and $\phi$ by the simple relations $A=\sqrt{{A_{\mathrm{c}}{ }^{2}+A_{\mathrm{s}}{ }^{2}}^{2}}$ and phase $\phi=\arctan \left(A_{\mathrm{c}} / A_{\mathrm{s}}\right)$. The time origin $\tau$ can be arbitrarily fixed, but the canonical choice is (Lomb 1976; Scargle 1982)

$\tan (2 \omega \tau)=\frac{\sum_{f=B, V, R, I} \sum_{j=1}^{N_{f}} \frac{\sin \left(\omega t_{f, j}\right)}{\sigma_{f, j}^{2}}}{\sum_{f=B, V, R, I} \sum_{j=1}^{N_{f}} \frac{\cos \left(\omega t_{f, j}\right)}{\sigma_{f, j}^{2}}}$

which will be our definition of $\tau$. Then, the free parameters involved in the minimisation are $P, M_{f, 0}$ for $f=V, R(B, I), A_{\mathrm{c}}$ and $A_{\mathrm{s}}$ or equivalently, $A$ and $\phi$. However, being interested to amplitude and not to the phase we marginalise our statistics over $\phi$.

A set of best possible combinations of $M_{f, 0}$ for $f=V, R$ $(B, I), A_{\mathrm{c}}$ and $A_{\mathrm{s}}$ for each given $P$ in a suitable range $\left[P_{\min }, P_{\max }\right]$ is obtained by minimising

$$
\begin{aligned}
& \chi^{2}(P)=\sum_{f=B, V, R, I} \sum_{j=1}^{N_{f}} \frac{\left(A_{\mathrm{c}} C_{f, j}(P, \tau)+A_{\mathrm{s}} S_{f, j}(P, \tau)+M_{f, 0}-m_{f, j}\right)^{2}}{\sigma_{f, j}^{2}} \\
& C_{f, j}(P, \tau)=\cos \left(\frac{2 \pi}{P}\left(t_{f, j}-\tau\right)\right) \\
& S_{f, j}(P, \tau)=\sin \left(\frac{2 \pi}{P}\left(t_{f, j}-\tau\right)\right)
\end{aligned}
$$

where $m_{f, j}$ are the magnitudes for filter $f$ measured at the times $t_{f, j}$ with associated errors $\sigma_{f, j}$ with $j=1,2, \ldots, N_{f}$ the index of $N_{f}$ measures obtained for the filter $f$. The minimisation is carried out analytically, with the $\tau$ defined in Eq. (4). This way the method becomes a generalisation of the floating average periodogram (Cumming et al. 1999; Cumming 2004) and reduces to it in the case in which magnitudes come from a single filter, while in the case of homoscedastic data and null zero points we return to the classical LS periodogram.

The sensitivity to noise as a function of $P$ is not constant and varies with time. Figure 8 represents the result for a Montecarlo designed to asses the sensitivity to noise of the periodogram in wide range of periods for Sycorax (upper frame) and Prospero (lower frame). The Montecarlo code generates simulated time series assuming the same time sampling of data, the same errors, normal distribution of errors and time independent expectations, and then computes the corresponding periodogram. Dots in the figure represents the realization of such periodograms, the fullline represents the Fourier transform of the time window. The sparseness of that causes a strong aliasing with diurnal periodicities. The plot is dominated by the prominent $24-\mathrm{h}$ diurnal peak, followed by the 12-h, 8-h, 6-h, 4-h, 2-h peaks of decreasing amplitude. It is evident that above a period of 20 -h the sensitivity of the periodogram to random errors increases rapidly. Then, our data set is best suited to detect periods below 20 - $\mathrm{h}$, or better, due to the presence of the 12-h peak, periods below 10-h.

Significant periodicities for $P<10$-h are likely present in the data if at least for one $P$ in the range, the squared amplitude $A^{2}(P)=A_{c}^{2}(P)+A_{s}^{2}(P)$ obtained by minimising Eq. (5), exceeds a critical value $A_{\text {crit }}^{2}$, which is fixed by determining the false alarm 
probability for the given $\left[P_{\min }, P_{\max }\right], P_{\mathrm{FA}}\left(A^{2}>A_{\text {crit }}^{2}\right)^{4}$. This interval has been sampled uniformly with a step size $\Delta P=0.025 \mathrm{~h}$ (the results do not depend much on the choice of the step size) and the $P_{\mathrm{FA}}$ as a function of $A_{\text {crit }}^{2}$ has been assessed. In determining the $P_{\mathrm{FA}}$ we exploit the fact that we want to calculate this probability for values of $A_{\text {crit }}^{2}$ for which $P_{\mathrm{FA}}$ is small. In this case,

$P_{\mathrm{FA}}\left(A^{2}>A_{\text {crit }}^{2}\right) \approx P_{\mathrm{FA}, 0} \exp \left(-A_{\text {crit }}^{2} / A_{\text {crit }, 0}^{2}\right)$

which is good for $P_{\mathrm{FA}}<0.3$, and with the parameters $P_{\mathrm{FA}, 0}$ and $A_{\text {crit }, 0}^{2}$ determined from Montecarlo simulations. The last two columns of Table 2 report the results of this generalised version of the LS periodogram. Again, for Sycorax and Prospero quite a significant periodical signal is detected. For Setebos the detection is marginal, while for Stephano and Trinculo no detection can be claimed at all. There are many reasons for the lack of detections of periodicity, despite random noise cannot account for the variability. Among them a lack in sensitivity, the fact that the period is outside the optimal search window, and that the light-curve cannot be described as a sinusoid. In conclusion, it is evident that a significant variability is present in most of these data sets and that for Sycorax and Prospero the July 2005 data suggests the possibility to construct a periodical light curve.

A period can be considered a good candidate if i.) $\chi^{2}(P)$ has a local minimum; ii.) the amplitude $A(P)$ of the associated sinusoid is significantly above the noise; iii.) the period $P$ is not affected in a significant manner by aliasing with the sampling window. Of course one has to consider the fact that one period may be preferred to another one just by chance. Random fluctuations may lead to a different selection of the best fit period. We assess this problem by generating random realizations of time series with expectation given by the measured values of each sample and $\sigma$ fixed by their Gaussian errors. For each generation the period producing the minimum $\chi^{2}(P)$ has been determined. The probability of selection of each $P, P_{\text {sel }}(P)$, has been then derived. We then add a criterion iv) that a period $P$ is selected as most likely if it has the maximum $P_{\text {sel }}(P)$. Table 3 reports the results of the fit. Note the difference in $\chi^{2}$ between the best fit with a sinusoid and the $\chi^{2}$ in Table 2 .

Before looking at the results, it has to be stressed that lightcurves can be either single peaked or double peaked. In the second case the rotation period will be twice the light-curve period. We do not have enough data to discriminate between these two cases, therefore the rotational periods of the observed objects could be twice the light-curve periods.

\section{Sycorax}

Figure 7 on the left is the periodogram for Sycorax. The $\chi^{2}$ suggests that the three periods $P \approx 3.6,3.1$ and 2.8 -h are favoured with a very high level of confidence $\left(P_{\mathrm{FA}}<10^{-8}\right)$. Bootstrap shows that the first period is the preferred one in about $96.6 \%$ of the simulations. The third period is chosen in less than $3.4 \%$ of the cases, the second one instead is chosen in less than $0.01 \%$ of the cases. For the best fit case we obtain also $B_{0}=21.676 \pm$ $0.013, V_{0}=20.849 \pm 0.005, R_{0}=20.276 \pm 0.003$ which are compatible with the weighted averages discussed in the next section and are fairly independent of $P$. The same holds for the "derotated colours" $B_{0}-V_{0}=0.828 \pm 0.014, V_{0}-R_{0}=$ $0.573 \pm 0.006$.

4 The $P_{\mathrm{FA}}\left(A>A_{\text {crit }}^{2}\right)$ is the probability that random errors are responsible for the occurrence of a peak of squared amplitude $A^{2}>A_{\text {crit }}^{2}$ in the interval $\left[P_{\min }, P_{\max }\right]$ of interest.
Table 3. Possible periods and amplitudes from fitting of Eq. (3). Column $1 \chi^{2}$ for fitting, Col. 2 the confidence level (CL), Col. 3 the best fit period in hours and its estimated internal uncertainty, Col. 4 the corresponding amplitudes and their uncertainties. Solutions are ordered with increasing $\chi^{2}$.

\begin{tabular}{|c|c|c|c|c|}
\hline \multicolumn{3}{|c|}{ Sycorax } & \multirow{2}{*}{$\begin{array}{c}P \\
{[\mathrm{~h}]}\end{array}$} & \multirow{2}{*}{$\begin{array}{c}A \\
{[\mathrm{mag}]}\end{array}$} \\
\hline \# & $x^{2}$ & $\mathrm{CL}$ & & \\
\hline 1 & 90.734 & - & $3.60 \pm 0.02$ & $0.067 \pm 0.004$ \\
\hline 2 & 134.88 & $2 \sigma$ & $2.70 \pm 0.03$ & $0.065 \pm 0.003$ \\
\hline 3 & 179.59 & $3 \sigma$ & $3.04 \pm 0.02$ & $0.051 \pm 0.010$ \\
\hline 4 & 180.66 & $3 \sigma$ & $3.13 \pm 0.02$ & $0.051 \pm 0.010$ \\
\hline \multicolumn{3}{|c|}{ Prospero } & $P$ & $A$ \\
\hline \# & $\chi^{2}$ & $\mathrm{CL}$ & {$[\mathrm{h}]$} & [mag] \\
\hline 1 & 27.288 & - & $4.551 \pm 0.040$ & $0.221 \pm 0.027$ \\
\hline 2 & 38.759 & $2 \sigma$ & $3.827 \pm 0.064$ & $0.201 \pm 0.029$ \\
\hline 3 & 53.286 & $3 \sigma$ & $5.760 \pm 0.100$ & $0.162 \pm 0.090$ \\
\hline 4 & 55.079 & $3 \sigma$ & $3.300 \pm 0.100$ & $0.121 \pm 0.090$ \\
\hline
\end{tabular}

\section{Prospero}

Figure 7 on the right shows the periodogram for Prospero. Given a safe dependence between the phase angle and magnitude is not established for these bodies, we just used the data taken on July 2005 to evaluate the periodogram. Four periods are allowed at a $5 \sigma$ confidence level (c.l.) for $P \approx 4.6,3.8,5.7$ and and $3.3 \mathrm{~h}$, respectively. Bootstrap shows that the first peak is the preferred one in about $91 \%$ of the simulations. The second peak is chosen in less than $6 \%$ of the cases, the other peaks instead are chosen in less than $3 \%$ of the cases. Comparing the periodogram with the spectral window it is evident how the secondary peaks are close to alias of the diurnal 24-h and, 12 -h periods. Removing a 24-h sinusoid from data before performing the fit depresses the 24-h peak, but not the 4.6-h peak. On the contrary the removal of the 4.6-h component strongly depresses the spectrum in the range $P \approx 3-8$-h. By fitting the first and second night data separately, and avoiding the implicit 24 -h periodicity, the preferred period is 4.3-h. Another way to filter the diurnal 24-h periodicity is to shift in time the lightcurve of the second night to overlap the lightcurve of the first. Even in this case periods between 3 and $5 \mathrm{~h}$ look favoured. The spectral window for the data shows a leakage corresponding to $P \approx 4.3 \mathrm{~h}$. A secular variation may introduce power at periods longer than $48 \mathrm{~h}$ which should leak power at $P \approx 4.3 \mathrm{~h}$. The periodogram for simulated data with a linear time dependence has a peak in the $P \approx 4.3-4.6 \mathrm{~h}$ region, but in order to have an amplitude in the periodogram of $0.2 \mathrm{mag}$ a peak-to-peak variation in the simulated data about seven or eight times larger than the peak-to-peak variation observed in real data is needed. Exclusion of $B$ and/or $I$ data, or fitting only the $R$ data does not significantly change the results. The same holds if we remove the three $R$ points with the largest errors. As a consequence, the data suggests $P \approx 4.6$-h with $A \approx 0.21$ mag. The lower frame of Fig. 7 represents the variations of $B, V, R$, and $I$ magnitudes folded over the best fit sinusoid. For the best fit case we obtain also $B_{0}=24.584 \pm 0.123, V_{0}=23.841 \pm 0.053$, $R_{0}=23.202 \pm 0.020, I_{0}=22.805 \pm 0.043$ which are compatible with the weighted averages discussed in the next section and are fairly independent of $P$. The same holds for the "derotated colours" $B_{0}-V_{0}=0.743 \pm 0.134, V_{0}-R_{0}=0.639 \pm 0.057$, $R_{0}-I_{0}=0.397 \pm 0.047$.

\section{Setebos, Stephano, and Trinculo}

For the other three bodies no strong evidence is found for a periodicity in the present data. However for Setebos the data may be considered suggestive of some periodicity with $P_{\mathrm{FA}} \approx$ $0.7 \%$, the preferred period being $P \approx 4.38 \pm 0.05 \mathrm{~h}$ with 

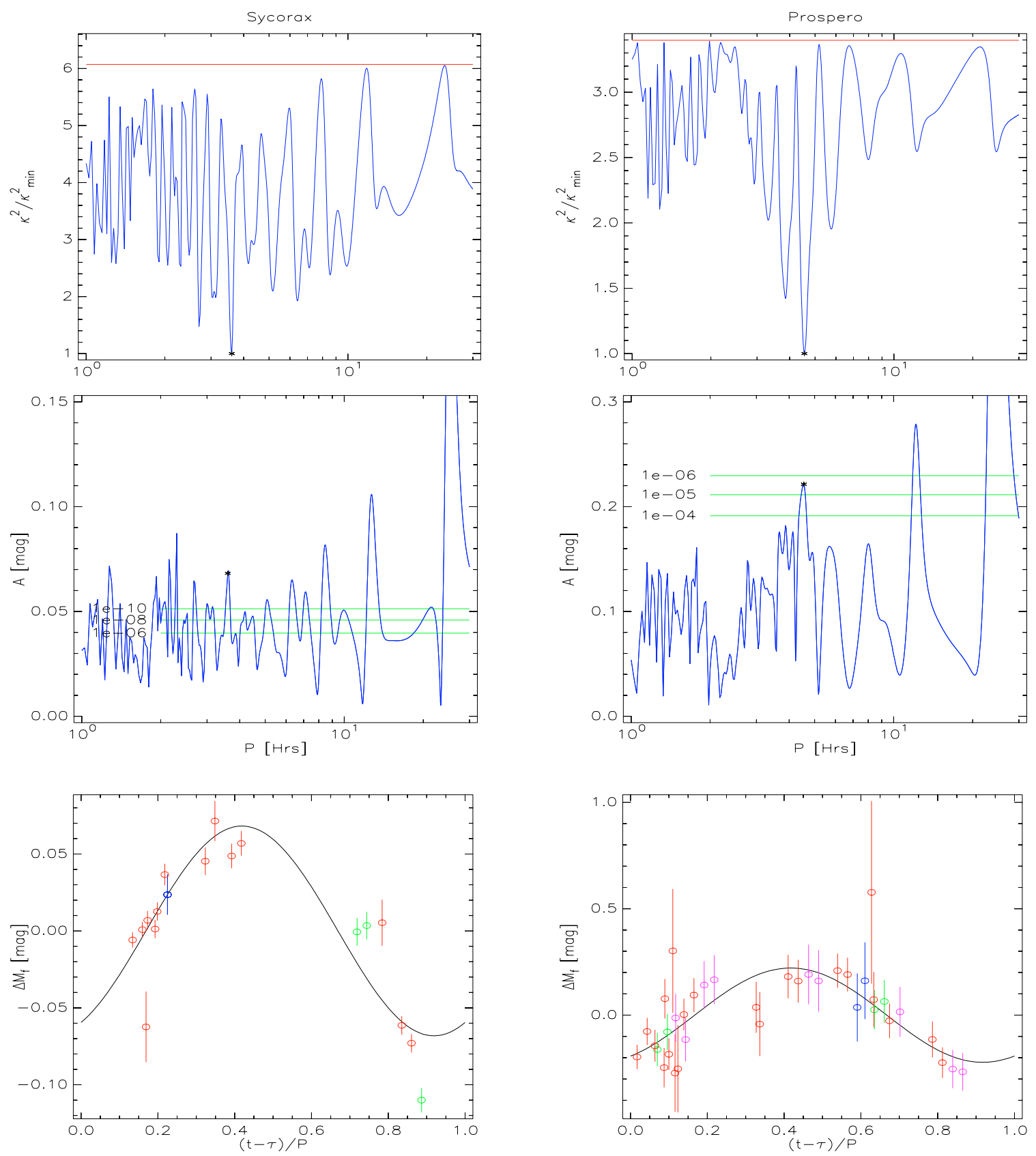

Fig. 7. LS test of periodicity for Sycorax (left) and Prospero (right) light-curve as a function of the period $P$. Upper frames display $\chi^{2} / \chi_{\min }^{2}$, the red line is the $\chi^{2} / \chi_{\min }^{2}$ for the case of a constant signal. The central frames are the estimated amplitude, a group of green lines denotes the amplitudes for $P_{\mathrm{FA}}=10^{-4}, 10^{-5}, 10^{-6}, 10^{-8}$ and $10^{-10}$. In all the frames a black $*$ marks the best fit point. Note that the diurnal 12-h and 24-h periods are excluded by the $\chi^{2} / \chi_{\min }^{2}$ despite the largest amplitude. The lower frames represents the overlap of the best reconstructed sinusoid with data ordered on a folded time scale, $(t-\tau) / P$, colours are blue for $B$, green for $V$, red for $R$, and magenta for $I$.

$A \approx 0.189 \pm 0.038 \mathrm{mag}$. For Stephano and Trinculo best fit periods are $P_{\text {Stephano }} \approx 2 \mathrm{~h}$ and $P_{\text {Trinculo }} \approx 5.7 \mathrm{~h}$ with amplitudes $A_{\text {Stephano }} \approx 0.459 \mathrm{mag}$ and $A_{\text {Trinculo }} \approx 0.422 \mathrm{~h}$ but with $P_{\mathrm{FA}}$ of $22 \%$ and $44 \%$, respectively.

\section{Averaged magnitudes and colours}

The derivation of colours would have to take into account the removal of rotational effects from magnitudes. Otherwise systematics as large as peak-to-peak variations in the light-curve can be expected. Lacking a good light-curve we may apply two possible methods: hierarchical determination of colours and comparison of weighted averages of magnitudes. The hierarchical method is based on the comparison of magnitudes from consecutive frames in the hypothesi that time differences are smaller than the lightcurve period, so that rotational effects can be neglected. An example is the estimation of Setebos $V-R$ by taking $V 3$ and $R 15$. In cases in which one of the frames obtained with a given filter $X$ is located between two frames of another filter $Y$, the $Y$ magnitude at the epoch of which the $X$ filter was acquired can be derived by simple linear interpolation. An example is given by the estimate of $V-R$ for Prospero in Nov. 22, by interpolating $R 2$ and $R 3$ at the epoch of $V 1$. Given in this way different estimates of each colours are obtained and weighted averages of such estimates are reported. The second method is based on the hypothesi that the light-curve has a periodical behaviour, and that it is so well 
Table 4. Weighted averages of magnitudes.

\begin{tabular}{lccccc}
\hline \hline Obj & Run & $B$ & $V$ & $R$ & $I$ \\
\hline Sycorax & Jul. & $21.701 \pm 0.013$ & $20.807 \pm 0.005$ & $20.276 \pm 0.002$ & \\
Prospero & Jul. & $24.678 \pm 0.120$ & $23.788 \pm 0.044$ & $23.160 \pm 0.019$ & $22.760 \pm 0.036$ \\
Prospero & Nov. & $24.651 \pm 0.115$ & $23.851 \pm 0.063$ & $23.196 \pm 0.028$ & $22.864 \pm 0.095$ \\
Setebos & Jul. & $24.455 \pm 0.132$ & $23.713 \pm 0.036$ & $23.192 \pm 0.018$ & \\
Stephano & Jul. & & $24.944 \pm 0.164$ & $24.212 \pm 0.050$ & \\
Trinculo & Jul. & & $25.670 \pm 0.413$ & $24.855 \pm 0.117$ & \\
\hline
\end{tabular}
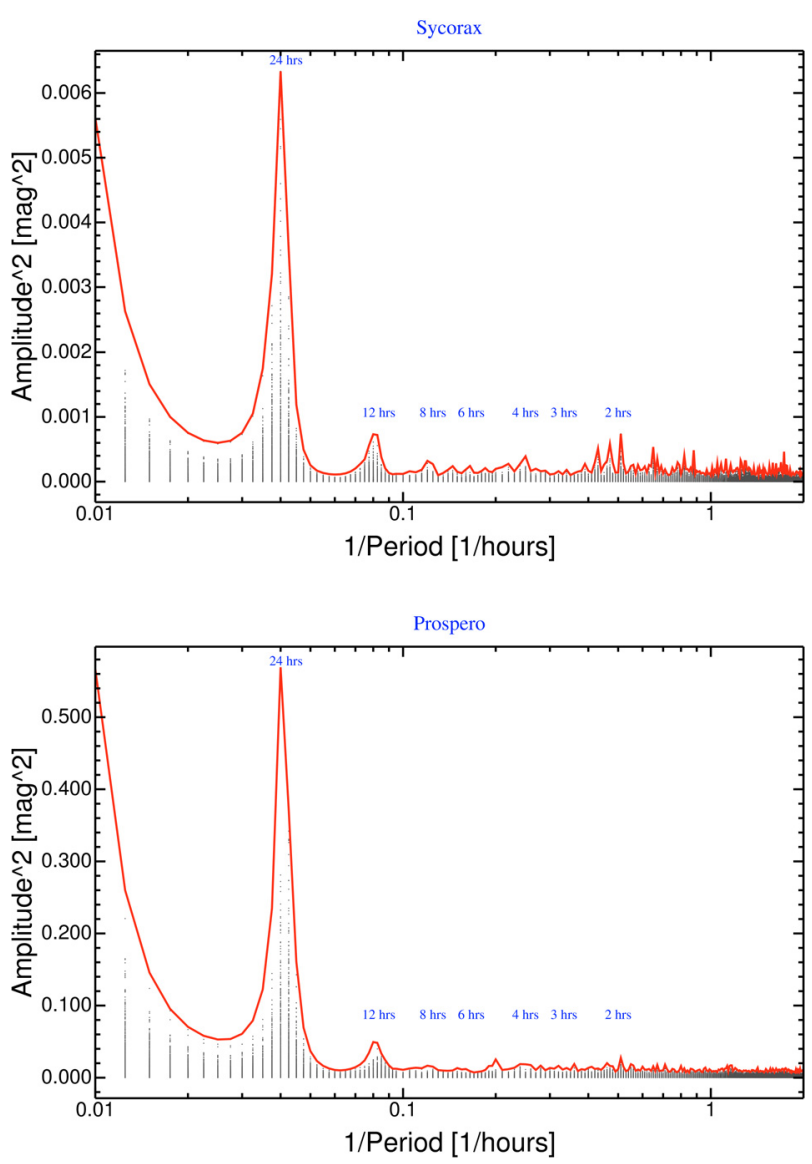

Fig. 8. Time window for Sycorax (upper frame) and Prospero (lower frame). (This figure is available in color in electronic form.)

sampled that weighting averages of magnitudes will cancel out the periodical time dependence. Of course the first method would be affected by larger random errors being based on a subset of the data. The second method is more prone to systematics.

Colours derived by using the first method for Sycorax, Prospero, and Setebos are presented in Table 5. When more independent estimates of the same colour are possible their weighted average is taken.

Colours derived from the second method are listed in Table 6 . The weighted averages of magnitudes for all of the satellites are listed in Table 4 . In both cases, tables report just random errors and not the systematic calibration error, which amounts to 0.018-0.022 mag, depending on the filter.

It is evident how the results of the two methods are similar. Hence, we present for conciseness the results of the second one as commonly reported in literature.
Table 5. Colours derived from the hierarchical method.

\begin{tabular}{lcccc}
\hline \hline Obj & Run & $B-V$ & $V-R$ & $R-I$ \\
\hline Sycorax & Jul. & $0.915 \pm 0.017$ & $0.548 \pm 0.006$ & \\
Prospero & Jul. & $0.793 \pm 0.138$ & $0.590 \pm 0.056$ & $0.395 \pm 0.046$ \\
Prospero & Nov. & $0.792 \pm 0.171$ & $0.635 \pm 0.085$ & $0.335 \pm 0.131$ \\
Setebos & Jul & $0.831 \pm 0.143$ & $0.444 \pm 0.053$ & \\
\hline
\end{tabular}

Table 6. Colours derived from weigthed averages.

\begin{tabular}{lcccc}
\hline \hline Obj & Run & $B-V$ & $V-R$ & $R-I$ \\
\hline Sycorax & Jul. & $0.893 \pm 0.014$ & $0.531 \pm 0.005$ & \\
Prospero & Jul. & $0.890 \pm 0.127$ & $0.628 \pm 0.048$ & $0.400 \pm 0.041$ \\
Prospero & Nov. & $0.800 \pm 0.131$ & $0.655 \pm 0.069$ & $0.332 \pm 0.099$ \\
Setebos & Jul. & $0.741 \pm 0.137$ & $0.522 \pm 0.041$ & \\
Stephano & Jul. & & $0.732 \pm 0.171$ & \\
Trinculo & Jul. & & $0.815 \pm 0.430$ & \\
\hline
\end{tabular}

\section{Sycorax}

We obtain $B-V=0.89 \pm 0.01$ and $V-R=0.53 \pm 0.01$, which are both compatible within $1 \sigma$ with the Maris et al. (2001) determinations but incompatible with Grav et al. (2004a).

\section{Prospero}

We have two sets of colour measures for Prospero, one from the July and the other from the November run. We obtain $B-V=$ $0.89 \pm 0.13, V-R=0.63 \pm 0.05$, and $R-I=0.40 \pm 0.04$ from the July run, and $B-V=0.85 \pm 0.13, V-R=0.59 \pm$ 0.07 and $R-I=0.33 \pm 0.10$. The two set are consistent. With respect to Grav et al. (2004a), we obtain a redder $V-R$, and a compatible $B-V$.

\section{Setebos}

We obtain $B-V=0.74 \pm 0.14$ and $V-R=0.52 \pm 0.04$. In this case Grav et al. (2004a) obtained their data with the Keck II telescope and DEIMOS, which hosts a rather special filter set. Our $B-V$ is compatible with their, while as for Prospero our $V-R$ is redder.

\section{Stephano and Trinculo}

For these two extremely faint satellites, we could only derive the $V-R$ colour, which is $0.73 \pm 0.17$ and $0.82 \pm 0.43$ for Stephano and Trinculo, respectively. While our $V-R$ for Trinculo is in good agreement with Grav et al. (2004a), their $V-R$ for Stephano is very low, and inconsistent with our one.

\section{Summary and conclusions}

In this paper we report accurate photometric $B, V, R, I$ observations obtained with the VLT telescope in two consecutive nights in July 2005 of the Uranian irregular satellites Sycorax, Stephano, Trinculo, and Setebos. Additional observations of Prospero obtained in November 22 and 25 of the same year are also reported. 
From the analysis of the data we conclude that Sycorax seems to display a variability of $\approx 0.07 \mathrm{mag}$, apparently larger than our previous result (Maris et al. 2001), while the period of 3.6-h is in agreement with our previous 2001 determination, and the same is true for the colours, so it seems unlikely that the difference in amplitude can be ascribed to some systematic. If true, a possible explanation would be that in the two epochs two different parts of the same light curve have been sampled. But also it has to be noted that larger brightness variations have been not reported by other observers in the past years (Gladman et al. 1998; Rettig et al. 2001; Romon et al. 2001). Prospero lightcurve exhibits an apparent periodicity of 4.6-h and an amplitude of $0.21 \mathrm{mag}$. The impact of such a sizeable amplitude is discussed throughly in (Parisi et al. 2007). Colours for Prospero obtained in July and November are in a quite good agreement, further assessing the correctness of the relative calibration. Setebos colours are only in marginal agreement with previous studies. In addition the Setebos light curve displays a significant variability but it is not possible to assess a good fit using a simple sinusoidal time dependence. Whether this is due to undersampling of the light-curve or to a non-sinusoidal time dependence can not be decided from these data alone. However, assuming a sinusoidal time dependence, our data are suggestive of a lightcurve amplitude of $\approx 0.18$ mag with a period of $\approx 4.4 \mathrm{~h}$ which will have to be confirmed or disproved by further observations. As for Stephano and Trinculo, the present data do not allow us to derive any sizeable time dependence, while the colours we derive are in marginal agreement with previous studies on the subject.

Acknowledgements. G.C. research is supported by Fundacion Andes. MGP research is supported by FONDAP (Centro de Astrofídica, Fondo de investigacíon avanzado en areas prioritarias). M.M. acknowledges FONDAP for financial support during a visit to Universidad the Chile. Part of the work of M.M. was also supported by INAF FFO - Fondo Ricerca Libera - 2006.

\section{References}

Agnor, C. B., \& Hamilton, D. P. 2006, Nature, 441, 192

Appenzeller, I., Fricke, K., Furtig, W., et al. 1998, The Messenger, 94, 1 Byl, J., \& Ovenden, M. W. 1975, MNRAS, 173, 579

Brunini, A., Parisi, M. G., \& Tancredi, G. 2002, Icarus, 159, 166

Carraro, G., Maris, M., Bertin D., \& Parisi, M. G. 2006, A\&A, 460, L39

Colombo, G., \& Franklin, F. A. 1971, Icarus, 15, 186

Connelly, R., \& Ostro, S. J. 1984, Geometriae Dedicata, 17, 87

Cumming, A. 2004, MNRAS, 354, 1165

Cumming, A., Marcy, G. W., \& Butler, R. P. 1999, ApJ, 526, 890

Gladman, B. J., Nicholson, P. D., Burns, J. A., et al. 1998, Nature, 392, 897

Gladman, B. J., Kavelaars, J. J., Holman, M. J., et al. 2000, Icarus, 147, 320

Grav, T., Holman, M. J., Brett, G., \& Kaare, A. 2003, Icarus, 166, 33

Grav, T., Holman, J., Matthew, J., \& Fraser Wesley, C. 2004a, ApJ, 613, L77

Grav, T., Holman, M. J., Gladman, B. J., \& Aksnes, K. 2004b, Icarus, 166, 33

Greenberg, R. J. 1976, in Jupiter, ed. T. Gehrels (Tucson: University of Arizona Press), 122

Heppenheimer, T. A. 1975, Icarus, 24, 172

Heppenheimer, T. A., \& Porco, C. 1977, Icarus, 30, 385

Horedt, G. P. 1976, AJ, 81, 675

Horne, J. H., \& Baliunas, S. L. 1986, ApJ, 302, 757

Jewitt, D., \& Sheppard, S. 2005, Space Sci. Rev., 116, 441

Kaasalinen, M., \& Torppa, J. 2001, Icarus, 153, 24

Kavelaars, J. J., Holman, M. J., Grav, T., et al. 2004, Icarus, 169, 474

Landolt, A. U. 1992, AJ, 104, 372

Lomb, N. R. 1976, Ap\&SS, 39, 447

Luu, J., \& Jewitt, D. 1996, AJ, 112, 2310

Maris, M., Carraro, G., Cremonese, G., \& Fulle, M. 2001, AJ, 121, 2800

Morrison, D., \& Burns, J. A. 1976, in Jupiter, ed. T. Gehrels (Tucson: University of Arizona Press), 991

Morrison, D., Cruikshank, D. P., \& Burns, J. A. 1977, in Planetary Satellites, ed. J. A. Burns (Tucson: University of Arizona Press), 3

Nesvorný, D., Alvarellos, J. L. A., Dones, L., \& Levison, H. F. 2003, AJ, 126, 398

Parisi, G., \& Brunini, A. 1997, Planet. Space Sci., 45, 181

Parisi, G., Carraro, G., Maris, M., \& Brunini, A. 2007, in preparation

Pollack, J. M., Burns, J. A., \& Tauber, M. E. 1979, Icarus, 37, 587

Rettig, T. V., Walsh, K., \& Consolmagno, G. 2001, Icarus, 154, 313

Romon, J., de Bergh, C., Barucci, M. A., et al. 2001, A\&A, 376, 310

Scargle, J. D. 1982, ApJ, 263, 835

Sheppard, S., Jewitt, D., \& Kleyna, J. 2005, AJ, 129, 518

Slattery, W. L., Benz, W., \& Cameron, A. G. W. 1992, Icarus, 99, 167

Tsui, K. H. 2000, Icarus, 148, 149 
M. Maris et al.: VLT Photometry of 5 Uranian Irregulars, Online Material p 1

\section{Online Material}


M. Maris et al.: VLT Photometry of 5 Uranian Irregulars, Online Material $p 2$

Table 1. Log of observations. U.T. refers to the start times of exposures and are not corrected for light travel time. The Flt. column refers to the filter $(B V R I)$ and frame number obtained with that filter (e.g.: $R 3$ is the third $R$ frame for the given object in the serie). $T_{\text {exp }}$ is the exposure time in seconds, the shortest exposures have been acquired to improve frame centering.

\begin{tabular}{|c|c|c|c|c|c|}
\hline Obj & Epoch & U.T. & Flt. & $\begin{array}{c}T_{\exp } \\
{[\mathrm{s}]}\end{array}$ & mag \\
\hline Sycorax & Jul. 29 & $03: 27: 13$ & $R 1$ & 30 & $20.282 \pm 0.015$ \\
\hline$"$ & " & $04: 40: 37$ & $R 2$ & 300 & $20.270 \pm 0.005$ \\
\hline$"$ & " & $04: 46: 05$ & $R 3$ & 300 & $20.277 \pm 0.005$ \\
\hline$"$ & " & $06: 46: 49$ & $V 1$ & 300 & $20.849 \pm 0.009$ \\
\hline " & " & $06: 52: 17$ & $V 2$ & 300 & $20.853 \pm 0.009$ \\
\hline$"$ & " & $08: 29: 17$ & $R 4$ & 300 & $20.277 \pm 0.006$ \\
\hline$"$ & " & $08: 34: 45$ & $R 5$ & 300 & $20.313 \pm 0.007$ \\
\hline " & " & $09: 12: 19$ & $R 6$ & 300 & $20.325 \pm 0.008$ \\
\hline$"$ & " & $09: 17: 47$ & $R 7$ & 300 & $20.333 \pm 0.008$ \\
\hline Sycorax & Jul. 30 & $02: 26: 25$ & $R 8$ & 30 & $20.214 \pm 0.023$ \\
\hline & & $06: 01: 03$ & $R 9$ & 300 & $20.283 \pm 0.006$ \\
\hline$"$ & " & $06: 06: 29$ & $R 10$ & 300 & $20.289 \pm 0.006$ \\
\hline$"$ & " & $06: 12: 15$ & $B 1$ & 300 & $21.701 \pm 0.013$ \\
\hline$"$ & " & $08: 24: 01$ & $R 11$ & 300 & $20.215 \pm 0.006$ \\
\hline$"$ & " & $08: 29: 28$ & $R 12$ & 300 & $20.203 \pm 0.006$ \\
\hline$"$ & " & $08: 35: 07$ & $V 3$ & 300 & $20.739 \pm 0.008$ \\
\hline$"$ & $"$ & $10: 09: 30$ & $R 13$ & 300 & $20.322 \pm 0.009$ \\
\hline$"$ & " & $10: 14: 56$ & $R 14$ & 300 & $20.348 \pm 0.013$ \\
\hline Stephano & Jul. 29 & $03: 34: 06$ & $R 1$ & 60 & $23.502 \pm 0.183$ \\
\hline " & & $03: 35: 34$ & $R 2$ & 60 & $23.960 \pm 0.297$ \\
\hline$"$ & " & $04: 15: 10$ & $R 3$ & 300 & $25.419 \pm 0.262$ \\
\hline$"$ & $"$ & $04: 20: 37$ & $R 4$ & 300 & $24.414 \pm 0.242$ \\
\hline$"$ & " & $04: 26: 04$ & $R 5$ & 300 & $24.271 \pm 0.210$ \\
\hline$"$ & " & $04: 31: 31$ & $R 6$ & 300 & $24.177 \pm 0.195$ \\
\hline$"$ & " & $06: 16: 34$ & $V 1$ & 600 & $25.033 \pm 0.269$ \\
\hline$"$ & " & $06: 27: 02$ & $V 2$ & 600 & $24.823 \pm 0.295$ \\
\hline$"$ & $"$ & $08: 03: 46$ & $R 7$ & 300 & $24.827 \pm 0.393$ \\
\hline$"$ & " & $08: 09: 14$ & $R 8$ & 300 & $24.538 \pm 0.299$ \\
\hline " & $"$ & $08: 14: 41$ & $R 9$ & 300 & $24.211 \pm 0.207$ \\
\hline$"$ & " & $08: 20: 09$ & $R 10$ & 300 & $24.554 \pm 0.291$ \\
\hline Stephano & Jul. 30 & $05: 35: 44$ & $R 11$ & 300 & $24.190 \pm 0.166$ \\
\hline & " & $05: 41: 11$ & $R 12$ & 300 & $24.119 \pm 0.157$ \\
\hline " & " & $05: 46: 39$ & $R 13$ & 300 & $24.056 \pm 0.148$ \\
\hline , & " & $05: 52: 07$ & $R 14$ & 300 & $24.114 \pm 0.151$ \\
\hline$"$ & " & $07: 57: 58$ & $R 15$ & 600 & $24.302 \pm 0.196$ \\
\hline , & 'I & $08: 08: 36$ & $V 3$ & 600 & $24.957 \pm 0.290$ \\
\hline " & "I & $09: 46: 41$ & $R 16$ & 600 & $24.285 \pm 0.269$ \\
\hline$"$ & $"$ & $09: 57: 11$ & $R 17$ & 600 & $24.276 \pm 0.250$ \\
\hline Setebos & Jul. 29 & $03: 05: 18$ & $R 1$ & 60 & $23.230 \pm 0.176$ \\
\hline & & $03: 54: 07$ & $R 2$ & 300 & $23.274 \pm 0.088$ \\
\hline$"$ & $" 1$ & $03: 59: 35$ & $R 3$ & 300 & $23.337 \pm 0.092$ \\
\hline$"$ & "' & $04: 05: 03$ & $R 4$ & 300 & $23.383 \pm 0.092$ \\
\hline$"$ & " & $05: 43: 04$ & $V 1$ & 600 & $23.796 \pm 0.080$ \\
\hline$"$ & " & $05: 53: 32$ & $V 2$ & 600 & $23.768 \pm 0.083$ \\
\hline " & " & $06: 03: 60$ & $V 3$ & 600 & $23.781 \pm 0.088$ \\
\hline$"$ & " & $07: 29: 43$ & $R 5$ & 600 & $23.196 \pm 0.080$ \\
\hline " & " & $07: 40: 12$ & $R 6$ & 600 & $23.242 \pm 0.088$ \\
\hline$"$ & " & $07: 50: 40$ & $R 7$ & 600 & $23.336 \pm 0.099$ \\
\hline$"$ & $"$ & $09: 25: 31$ & $R 8$ & 600 & $23.100 \pm 0.092$ \\
\hline$"$ & " & $09: 35: 58$ & $R 9$ & 600 & $22.847 \pm 0.074$ \\
\hline$"$ & " & $09: 46: 25$ & $R 10$ & 600 & $22.903 \pm 0.077$ \\
\hline
\end{tabular}

Table 1. continued.

\begin{tabular}{|c|c|c|c|c|c|}
\hline Obj & Epoch & U.T. & Flt. & $\begin{array}{c}T_{\exp } \\
{[\mathrm{s}]}\end{array}$ & mag \\
\hline Setebos & Jul. 30 & $02: 16: 30$ & $R 11$ & 60 & $23.836 \pm 0.318$ \\
\hline$"$ & " & $05: 01: 08$ & $R 12$ & 600 & $23.272 \pm 0.064$ \\
\hline$"$ & " & $05: 11: 37$ & $R 13$ & 600 & $23.182 \pm 0.062$ \\
\hline$"$ & $"$ & $05: 22: 04$ & $R 14$ & 600 & $23.314 \pm 0.068$ \\
\hline$"$ & $"$ & $07: 02: 39$ & $R 15$ & 600 & $23.127 \pm 0.061$ \\
\hline ” & $"$ & $07: 13: 06$ & $R 16$ & 600 & $23.205 \pm 0.066$ \\
\hline$"$ & " & $07: 23: 51$ & $B 1$ & 600 & $24.455 \pm 0.132$ \\
\hline$"$ & $"$ & $07: 34: 30$ & $V 4$ & 600 & $23.649 \pm 0.081$ \\
\hline$"$ & $"$ & $07: 44: 58$ & $V 5$ & 600 & $23.602 \pm 0.075$ \\
\hline " & " & $09: 13: 23$ & $R 17$ & 600 & $23.223 \pm 0.077$ \\
\hline$"$ & $"$ & $09: 23: 50$ & $R 18$ & 600 & $23.219 \pm 0.078$ \\
\hline " & " & $09: 34: 18$ & $R 19$ & 600 & $23.249 \pm 0.084$ \\
\hline Trinculo & Jul. 29 & $04: 15: 10$ & $R 3$ & 300 & $25.110 \pm 0.474$ \\
\hline & " & $04: 20: 37$ & $R 4$ & 300 & $24.862 \pm 0.378$ \\
\hline$"$ & " & $04: 26: 04$ & $R 5$ & 300 & $24.544 \pm 0.272$ \\
\hline$"$ & " & $04: 31: 31$ & $R 6$ & 300 & $25.222 \pm 0.485$ \\
\hline$"$ & " & $06: 16: 34$ & $V 1$ & 600 & $25.925 \pm 0.671$ \\
\hline$"$ & $"$ & $06: 27: 02$ & $V 2$ & 600 & $26.189 \pm 0.901$ \\
\hline$"$ & $"$ & $08: 03: 46$ & $R 7$ & 300 & $24.711 \pm 0.362$ \\
\hline$"$ & $"$ & $08: 09: 14$ & $R 8$ & 300 & $25.237 \pm 0.568$ \\
\hline " & $"$ & $08: 14: 41$ & $R 9$ & 300 & $24.626 \pm 0.330$ \\
\hline$"$ & $"$ & $08: 20: 09$ & $R 10$ & 300 & $24.780 \pm 0.361$ \\
\hline Trinculo & Jul. 30 & $05: 35: 44$ & $R 11$ & 300 & $25.559 \pm 0.631$ \\
\hline & " & $05: 41: 11$ & $R 12$ & 300 & $25.664 \pm 0.713$ \\
\hline$"$ & " & $05: 46: 39$ & $R 13$ & 300 & $24.996 \pm 0.386$ \\
\hline " & " & $05: 52: 07$ & $R 14$ & 300 & $25.988 \pm 0.976$ \\
\hline " & " & $07: 57: 58$ & $R 15$ & 600 & $24.550 \pm 0.465$ \\
\hline$"$ & " & $08: 08: 36$ & $V 3$ & 600 & $25.167 \pm 0.646$ \\
\hline Prospero & Jul. 29 & $03: 29: 44$ & $R 1$ & 100 & $23.275 \pm 0.130$ \\
\hline " & " & $03: 39: 16$ & $R 2$ & 300 & $23.175 \pm 0.082$ \\
\hline$"$ & " & $03: 46: 44$ & $I 1$ & 300 & $22.821 \pm 0.117$ \\
\hline$"$ & " & $05: 12: 00$ & $R 3$ & 400 & $23.005 \pm 0.057$ \\
\hline " & " & $05: 19: 09$ & $R 4$ & 400 & $23.125 \pm 0.064$ \\
\hline$"$ & " & $05: 26: 27$ & $V 1$ & 400 & $23.680 \pm 0.079$ \\
\hline$"$ & $"$ & $05: 33: 34$ & $V 2$ & 400 & $23.763 \pm 0.085$ \\
\hline " & " & $06: 39: 14$ & $R 5$ & 100 & $23.239 \pm 0.120$ \\
\hline ” & $"$ & $06: 42: 09$ & $R 6$ & 50 & $23.160 \pm 0.150$ \\
\hline " & " & $06: 59: 28$ & $R 7$ & 400 & $23.383 \pm 0.102$ \\
\hline$"$ & $"$ & $07: 06: 36$ & $R 8$ & 400 & $23.362 \pm 0.100$ \\
\hline$"$ & " & $07: 13: 55$ & $I 2$ & 400 & $22.996 \pm 0.140$ \\
\hline$"$ & " & $07: 21: 03$ & I3 & 400 & $22.966 \pm 0.144$ \\
\hline$"$ & " & $08: 41: 56$ & $R 9$ & 400 & $23.087 \pm 0.084$ \\
\hline$"$ & " & $08: 49: 04$ & $R 10$ & 400 & $22.979 \pm 0.072$ \\
\hline$"$ & $"$ & $08: 56: 23$ & $I 4$ & 400 & $22.552 \pm 0.090$ \\
\hline$"$ & $"$ & $09: 03: 30$ & I5 & 400 & $22.539 \pm 0.089$ \\
\hline
\end{tabular}


M. Maris et al.: VLT Photometry of 5 Uranian Irregulars, Online Material p 3

Table 1. continued.

\begin{tabular}{|c|c|c|c|c|c|}
\hline Obj & Epoch & U.T. & Flt. & $\begin{array}{c}T_{\exp } \\
{[\mathrm{s}]}\end{array}$ & mag \\
\hline Prospero & Jul. 30 & $02: 13: 32$ & $R 11$ & 60 & $23.779 \pm 0.429$ \\
\hline & " & $04: 18: 45$ & $R 12$ & 98 & $22.955 \pm 0.092$ \\
\hline$"$ & " & $04: 21: 15$ & $R 13$ & 210 & $23.018 \pm 0.074$ \\
\hline$"$ & " & $04: 25: 37$ & $R 14$ & 20 & $23.504 \pm 0.291$ \\
\hline$"$ & " & $04: 27: 11$ & $R 15$ & 20 & $22.929 \pm 0.182$ \\
\hline " & " & $04: 29: 12$ & $R 16$ & 20 & $22.949 \pm 0.205$ \\
\hline " & " & $04: 30: 18$ & $R 17$ & 400 & $23.205 \pm 0.075$ \\
\hline " & $"$ & $04: 37: 26$ & $R 18$ & 400 & $23.296 \pm 0.080$ \\
\hline " & $\prime \prime$ & $04: 44: 43$ & $I 6$ & 400 & $22.947 \pm 0.112$ \\
\hline " & " & $04: 51: 51$ & $I 7$ & 400 & $22.972 \pm 0.114$ \\
\hline " & " & $06: 19: 32$ & $R 19$ & 400 & $23.411 \pm 0.080$ \\
\hline " & " & $06: 26: 39$ & $R 20$ & 400 & $23.392 \pm 0.079$ \\
\hline$"$ & " & $06: 34: 20$ & $B 1$ & 300 & $24.622 \pm 0.160$ \\
\hline " & " & $06: 39: 48$ & $B 2$ & 300 & $24.748 \pm 0.180$ \\
\hline " & " & $06: 45: 40$ & $V 3$ & 400 & $23.867 \pm 0.092$ \\
\hline$"$ & " & $06: 52: 47$ & $V 4$ & 400 & $23.906 \pm 0.101$ \\
\hline$"$ & " & $08: 42: 41$ & $R 21$ & 400 & $23.057 \pm 0.075$ \\
\hline$"$ & " & $08: 49: 48$ & $R 22$ & 400 & $23.279=$ \\
\hline " & " & $08: 57: 25$ & 18 & 400 & 0.113 \\
\hline ” & " & $09: 04: 32$ & 19 & 400 & $22.690=$ \\
\hline Prospero & Nov. 22 & $00: 44: 18$ & $R 1$ & 300 & $23.193=$ \\
\hline & & $00: 50: 04$ & $B 1$ & 300 & $24.583 \pm 0.181$ \\
\hline " & " & $00: 55: 49$ & $R 2$ & 300 & $23.240 \pm 0.081$ \\
\hline " & " & $01: 01: 27$ & $V 1$ & 300 & $23.881 \pm 0.110$ \\
\hline$"$ & $"$ & $01: 07: 06$ & $R 3$ & 300 & $23.310 \pm 0.090$ \\
\hline$"$ & " & $01: 12: 45$ & $I 1$ & 300 & $22.726 \pm 0.157$ \\
\hline$"$ & " & $01: 15: 43$ & $I 2$ & 300 & $22.925 \pm 0.243$ \\
\hline$"$ & " & $01: 18: 52$ & $R 4$ & 300 & $23.120 \pm 0.076$ \\
\hline$"$ & " & $01: 24: 38$ & $B 2$ & 300 & $24.710 \pm 0.218$ \\
\hline$"$ & " & $01: 30: 25$ & $R 5$ & 300 & $23.212 \pm 0.083$ \\
\hline Prospero & Nov. 25 & $00: 36: 16$ & $V 2$ & 300 & $23.835 \pm 0.109$ \\
\hline & & $00: 41: 54$ & $R 6$ & 300 & $23.194 \pm 0.080$ \\
\hline$"$ & " & $00: 47: 32$ & $I 3$ & 300 & $22.838 \pm 0.184$ \\
\hline$"$ & $"$ & $00: 50: 29$ & $I 4$ & 300 & $22.969 \pm 0.207$ \\
\hline ” & $"$ & $00: 53: 37$ & $R 7$ & 300 & $23.279 \pm 0.088$ \\
\hline ” & $" \prime$ & $00: 59: 23$ & $B 3$ & 300 & $24.686 \pm 0.203$ \\
\hline$"$ & " & $01: 05: 07$ & $R 8$ & 300 & $23.063 \pm 0.076$ \\
\hline$"$ & " & $01: 10: 45$ & $V 3$ & 300 & $23.837 \pm 0.108$ \\
\hline$"$ & $"$ & $01: 16: 22$ & $R 9$ & 300 & $23.284 \pm 0.165$ \\
\hline
\end{tabular}

